\title{
Atitudes dos trabalhadores de saúde mental em relação aos pacientes psiquiátricos em uma cidade do interior do Estado do Rio de Janeiro
}

\author{
Attitudes of mental health workers' toward mentally ill \\ patients in a small city situated in the Rio de Janeiro state
}

\author{
Ludmila Abramenkoํㅜ, Giovanni Marco Lovisi ${ }^{1}$, Diego de Lima Fonseca ${ }^{1}$, \\ Lucia Abelha ${ }^{1}$
}

\begin{abstract}
Resumo
Introdução: Existem poucos estudos acerca da avaliação das atitudes dos trabalhadores em saúde mental em relação aos indivíduos com transtornos mentais no Brasil, principalmente em cidades de médio e pequeno porte. Portanto, foi conduzido um estudo seccional para avaliar as atitudes dos profissionais de saúde mental que trabalham em uma pequena cidade do interior do Estado do Rio de Janeiro. Método: Foram avaliados 155 trabalhadores em saúde mental do município de Carmo, em novembro de 2015, utilizando a escala Community Attitudes Toward Mentally III (CAMI-BR). A CAMI tem quatro subescalas: Autoritarismo, Benevolência, Restrição Social e Ideologia de Saúde Mental Comunitária. Resultados: Os trabalhadores, na sua maioria, apresentaram atitudes positivas para com os pacientes psiquiátricos. A subescala que teve pontuação mais alta foi Benevolência, seguida por Ideologia Comunitária, enquanto Restrição Social e Autoritarismo obtiveram as pontuações mais baixas. As variáveis que tiveram associação significativa com as atitudes positivas foram: faixa etária, categoria profissional e nível educacional. As pessoas mais jovens e com mais anos de estudo foram as que mostraram atitudes mais positivas. Os Cuidadores apresentaram atitudes mais negativas quando comparados às outras categorias. Conclusão: Embora ainda persistam algumas atitudes autoritárias no cuidado dos pacientes psiquiátricos, os trabalhadores de Carmo têm atitudes positivas relacionadas às pessoas com transtorno mental e sua inclusão na comunidade, o que fortalece a implementação das reformas da assistência psiquiátrica no município.
\end{abstract}

Palavras-chave: estigma social; transtornos mentais; atitude; desinstitucionalização.

\begin{abstract}
Introduction: As there are few studies about evaluation of attitudes of mental health workers towards individuals with mental disorders in Brazil, especially in small and medium-sized towns. A cross-sectional study was carried out to assess attitudes of mental health professionals working in a small city situated in the Rio de Janeiro State. Methods: Data were collected using the Community Attitudes Towards Mentally III (CAMI-BR) in a population of mental health professionals working in Carmo in November 2015. The CAMI consists of four subscales: Authoritarianism, Benevolence, Social Restrictiveness and Community Mental Health Ideology. Results: Most workers presented positive attitudes towards psychiatric patients. The subscale with the highest score was Benevolence followed by Community Ideology, while Social Restriction and Authoritarianism obtained the lowest scores. The following individual characteristics were associated with positive attitudes: age group, professional category and educational level, being the youngest people with higher level education showing more positive attitudes. The caregivers presented more negative attitudes when compared to the other categories. Conclusion: Although there are still some authoritarian professionals' attitudes in the care of psychiatric patients in Carmo, these workers have positive attitudes related to the treatment of people with mental disorders and their inclusion in the community, which strengthens the implementation of psychiatric care reforms in the town.
\end{abstract}

Keywords: social stigma; mental disorders; attitude; deinstitutionalization.

IInstituto de Estudos em Saúde Coletiva (IESC), Universidade Federal do Rio de Janeiro (UFRJ) - Rio de Janeiro (RJ), Brasil.

Trabalho realizado no Instituto de Estudos em Saúde Coletiva (IESC), Universidade Federal do Rio de Janeiro (UFRJ) - Rio de Janeiro (RJ), Brasil.

Endereço para correspondência: Lucia Abelha - Instituto de Estudos em Saúde Coletiva (IESC), Universidade Federal do Rio de Janeiro (UFRJ), Avenida Horácio Macedo, s/n - Ilha do Fundão - CEP: 21944-970 - Rio de Janeiro (RJ), Brasil - Email: abelha@iesc.ufrj.br

Fonte de financiamento: Bolsa CAPES.

Conflito de interesses: nada a declarar. 


\section{INTRODUÇÃO}

Para que a desinstitucionalização psiquiátrica tenha o sucesso esperado, é necessário que a comunidade ofereça o apoio e um ambiente favorável para que os indivíduos com transtornos mentais tenham os mesmos direitos que qualquer outro cidadão. As atitudes negativas, causadas frequentemente pelo estigma da doença, são algumas das principais barreiras para a inclusão social dessa população na comunidade. O estigma afeta a continuidade do tratamento, dificulta as relações familiares, provoca o isolamento social e a perda da autoestima, contribuindo para o impedimento da instalação de serviços de saúde mental nas comunidades ${ }^{1-3}$.

Os profissionais de saúde mental - importantes instrumentos de trabalho e principal tecnologia na área - são o primeiro contato do paciente com os serviços. Por isso, é de fundamental importância conhecer as atitudes positivas ou negativas desses trabalhadores em relação aos pacientes para saber os pontos relevantes que dificultam a inclusão destes indivíduos na sociedade.

Esses profissionais possuem uma posição estratégica por sua proximidade com a população assistida. Esse contato é capaz de produzir profundos efeitos na formação de atitudes em relação às pessoas com transtornos mentais, já que inclui não apenas $o$ aspecto cognitivo, mas também o afetivo e comportamental das relações interpessoais. Assim sendo, os trabalhadores de saúde mental têm um papel fundamental na vida dos usuários e são, muitas vezes, o maior apoio no percurso da inclusão social ${ }^{4,5}$.

Algumas pesquisas mostram uma atitude negativa dos profissionais de saúde, quando comparados à população geral, para com indivíduos que possuem transtornos mentais, como os estudos de Nordt et al. ${ }^{6}$, Thorpe e Addison ${ }^{7}$ e Lauber et al. ${ }^{8}$, nos quais as variáveis sociodemográficas que mais influenciaram as atitudes negativas dos profissionais em saúde mental foram idade, sexo, nível educacional e renda. O grupo socioeconômico mais baixo, de menor nível educacional, e as pessoas de minoria étnicas apresentaram atitudes mais negativas em relação a esse grupo.

Outros estudos, ao contrário, demonstraram que os profissionais de saúde mental apresentaram atitudes mais positivas para com os doentes mentais - o contato direto foi um importante fator para isso - do que a população geral ${ }^{5,9-11}$.

No Brasil, há poucos estudos que avaliam as atitudes de profissionais de saúde mental com relação aos indivíduos com transtornos mentais. Loch ${ }^{12}$ pesquisaram as atitudes dos psiquiatras para com esquizofrênicos no Congresso Brasileiro de Psiquiatria (2009) em São Paulo. Para tanto, utilizaram um questionário que avaliava estereótipos negativos, opinião sobre psicotrópicos e tolerância aos efeitos colaterais. Os autores encontraram que os psiquiatras tinham, na sua maioria, estereótipos negativos em relação às pessoas com esquizofrenia e atitudes positivas quanto ao uso e à tolerância aos efeitos colaterais de psicotrópicos. Siqueira et al. ${ }^{5}$, que avaliaram as atitudes de profissionais de saúde em geral de um hospital universitário no Rio de Janeiro, encontraram que os trabalhadores com maior nível de escolaridade e maior experiência profissional apresentaram atitudes mais positivas.

Há escassez de trabalhos no Brasil, principalmente nos municípios de pequeno e médio porte, onde os novos dispositivos de atenção na comunidade foram recentemente implementados. Portanto, o objetivo do presente estudo é avaliar as atitudes dos trabalhadores da área de saúde mental do município de Carmo no Estado de Rio de Janeiro.

\section{MÉTODOS}

\section{Local do estudo}

O estudo foi realizado com os trabalhadores de saúde mental do município de Carmo, localizado na Região Serrana do Estado de Rio de Janeiro, com uma população de 18.322 habitantes ${ }^{13}$.

Em 1947, foi criado em Carmo um hospital colônia para pacientes psiquiátricos, o qual chegou a ter 400 pacientes homens que vieram de outros municípios e/ou transferidos de outros hospitais do Estado do Rio de Janeiro e Minas Gerais, como o Hospital Psiquiátrico de Jurujuba e Vargem Alegre. Até 1968, o hospital ainda fazia uso de castigos, como os quartos fortes ou cubículos e o isolamento dos indivíduos. A partir de então, a estrutura mudou, havendo a retirada das grades e a intensificação de atividades agrícolas e pecuárias. Entretanto, as condições de abandono e descuido com os pacientes persistiram, até que, no ano de 2001, a Secretaria Estadual de Saúde do Rio de Janeiro iniciou um processo de intervenção na instituição, tendo em vista a redução dos leitos e o fechamento do hospital, que contava com 280 pacientes internados. Com isso, iniciou-se o processo de saída de todos eles do hospital para a comunidade. Atualmente, no município, existem 21 residências terapêuticas para 122 moradores. A Rede de Saúde Mental da cidade é composta por diferentes dispositivos: Centro de Atenção Psicossocial (CAPS), Equipe de Saúde Mental no Hospital Geral, Centro de Convivência e Centro de Atenção para Infância e Juventude.

\section{População do estudo}

O estudo abrangeu a totalidade dos trabalhadores da Rede de Saúde Mental do município. Eram 167 funcionários distribuídos nos seguintes serviços: 118 trabalhavam 24 horas em turnos rotativos em 21 Serviços Residenciais Terapêuticos (SRT), onde moravam aproximadamente oito pacientes em cada residência; oito, em um CAPS II; cinco, em um Centro de Convivência; seis, em quatro Leitos de Referência em Saúde Mental no Hospital Geral; e sete, em um Centro de Atenção para Infância e Juventude. As atividades da Rede eram coordenadas pelo Núcleo Estadual 
de Saúde Mental, no qual, além das atividades de coordenação e supervisão, realizavam-se atividades de ensino e pesquisa com 23 funcionários.

\section{Coleta de dados}

A coleta de dados foi realizada pela autora principal do trabalho, no mês de novembro de 2015. Após assinarem o Termo de Consentimento Livre e Esclarecido, os participantes receberam o questionário para autopreenchimento. Os seguintes instrumentos foram utilizados:

\section{Instrumentos de pesquisa}

\section{Atitudes da Comunidade em Relação aos Doentes Mentais (CAMI-BR)}

A Community Attitudes toward the Mentally Ill (CAMI), desenvolvida por Taylor e Dear ${ }^{14}$ e validada para o Brasil por Gonçalves et al. ${ }^{15}$, é uma escala multidimensional cujo objetivo principal é avaliar a atitude das comunidades para o estabelecimento de dispositivos de saúde mental.

A CAMI-BR é composta por 40 itens distribuídos em quatro subescalas, que avaliam os seguintes sentimentos:

Autoritarismo: a necessidade de internação hospitalar, a diferenciação entre pessoas com e sem transtorno mental, a importância do cuidado tutelar e as causas da doença mental.

Restrição Social: as opiniões sobre periculosidade do doente mental, a necessidade de distância social, a falta de responsabilidade e a normalidade do paciente psiquiátrico.

Benevolência: a responsabilidade da sociedade quanto aos transtornos psiquiátricos, a necessidade de atitudes de simpatia e cuidado, o desejo de se envolver pessoalmente e os sentimentos de benevolência.

Ideologia da Saúde Mental Comunitária: o impacto da implantação dos serviços de saúde mental na vizinhança, o perigo que o paciente psiquiátrico representa para os vizinhos e a aceitação dos princípios da desinstitucionalização psiquiátrica.

As respostas foram distribuídas em uma escala Likert, que variou de 0 a 4, em que: 0 - Concordo totalmente; 1 - Concordo; 2 - Tanto faz; 3 - Discordo; 4 - Discordo totalmente. Quanto maior o escore da escala, mais positiva é a atitude com as pessoas que possuem transtorno mental.

\section{Questionário sociodemográfico}

O questionário sociodemográfico foi construído a partir do instrumento utilizado por Wolff et al. ${ }^{16}$. As variáveis utilizadas foram: idade, sexo, estado civil, religião, imóvel (próprio ou alugado), nível educacional, categoria profissional, local de trabalho, faixa etária dos filhos e uso de serviços de saúde mental.

\section{Análise estatística}

Para a proporção de respostas positivas e negativas em cada item das quatro subescalas, foi feita uma recodificação da escala Likert:

$\mathbf{0} \mathbf{1}$-(Concordo fortemente e Concordo) - recodificado para 1.

2 - foi mantido o mesmo escore.

3 e 4 -(Discordo e Discordo fortemente - recodificado para 3.

$\mathrm{O}$ escore final da escala foi a média de cada subescala e variou de 0 a 4.

Para avaliar a relação entre cada subescala e as características da população estudada, utilizaram-se o t de Student (variáveis categóricas) e a Análise de Variância (ANOVA) (variáveis contínuas).

Uma análise multivariada de variância (MANOVA) foi feita para avaliar se as características individuais dos trabalhadores poderiam predizer suas atitudes em relação aos pacientes psiquiátricos nas quatro subescalas. Essa análise controla a correlação entre variáveis dependentes (subescalas) por meio de múltiplos testes de correlação ${ }^{17}$. Foram considerados significativos valores de p menor ou igual a 0,05 .

Para a digitação dos dados, foi utilizado o programa EpiInfo (versão 7), e para a análise dos dados, os programas SPSS (versão 16.0).

\section{Questões éticas}

O estudo foi aprovado pelo Comitê de Ética em Pesquisa do Instituto de Estudos em Saúde Coletiva da UFRJ, parecer $n^{\circ}$ 1.267.425. Todos os participantes da pesquisa assinaram o Termo de Consentimento Livre e Esclarecido.

\section{RESULTADOS}

Dos 167 trabalhadores de saúde mental do município, 155 responderam ao questionário, o que representa uma perda de 12 entrevistas (7,1\%). Os entrevistados eram, na sua maioria, mulheres (71,6\%), com idade compreendida entre 31 e 50 anos $(65,8 \%)$, casados $(60,6 \%)$, católicos $(55,5 \%)$, com imóvel próprio $(67,7 \%)$ e bom nível educacional (67,7\%), que não frequentavam nem conheciam alguém próximo com necessidade de serviços de saúde mental (59,4\%). A categoria profissional preponderante entre os entrevistados, como já esperado, foi a de Cuidador $(72,2 \%)$ e que desenvolviam suas funções nos SRT (Tabela 1).

O escore total da escala CAMI foi 3,05. A subescala Benevolência apresentou a maior pontuação $(3,27)$, seguida das subescalas Ideologia da Saúde Mental Comunitária $(3,24)$, Restrição Social $(3,05)$ e Autoritarismo $(2,65)$. Esses resultados demonstraram uma atitude que oscilou entre neutra (subescala Autoritarismo) e positiva (as demais). Na Tabela 2, podemos observar que os participantes tiveram atitudes positivas em relação aos pacientes na maioria dos itens. Apenas quatro itens apresentaram mais 
Tabela 1. Distribuição das características sociodemográficas da população estudada

\begin{tabular}{|c|c|c|}
\hline Variável & $\mathbf{n}$ & $\%$ \\
\hline \multicolumn{3}{|l|}{ Faixa etária } \\
\hline 20 a 30 anos & 20 & 12,9 \\
\hline 31 a 40 & 51 & 32,9 \\
\hline 41 a 50 & 51 & 32,9 \\
\hline 51 a 60 & 27 & 17,4 \\
\hline$>60$ & 6 & 3,9 \\
\hline \multicolumn{3}{|l|}{ Sexo } \\
\hline Masculino & 44 & 28,4 \\
\hline Feminino & 111 & 71,6 \\
\hline \multicolumn{3}{|l|}{ Estado Civil } \\
\hline Solteiro & 40 & 25,8 \\
\hline Casado/companheiro & 94 & 60,6 \\
\hline Separado & 5 & 3,3 \\
\hline Divorciado & 12 & 7,7 \\
\hline Viúvo & 4 & 2,6 \\
\hline \multicolumn{3}{|l|}{ Religião } \\
\hline Sem religião & 5 & 3,2 \\
\hline Católico & 86 & 55,5 \\
\hline Batista & 9 & 5,8 \\
\hline Universal & 2 & 1,3 \\
\hline Assembleia de Deus & 9 & 5,8 \\
\hline Cardecista & 6 & 3,9 \\
\hline Umbanda & 3 & 1,9 \\
\hline Outras & 33 & 21,3 \\
\hline S/Inf. & 2 & 1,3 \\
\hline \multicolumn{3}{|l|}{ Imóvel } \\
\hline Próprio & 105 & 67,7 \\
\hline Alugado & 44 & 28,4 \\
\hline S/Inf. & 6 & 3,9 \\
\hline \multicolumn{3}{|l|}{ Nível educacional } \\
\hline Até 9 anos de estudo & 50 & 32,2 \\
\hline 10 a 12 anos & 55 & 35,5 \\
\hline Mais de 12 anos & 48 & 31 \\
\hline S/Inf. & 2 & 1,3 \\
\hline \multicolumn{3}{|l|}{ Categoria profissional } \\
\hline Cuidadores & 112 & 72,25 \\
\hline Função de Nível Superior & 25 & 16,12 \\
\hline Administrativos & 12 & 7,74 \\
\hline Coordenação & 6 & 3,89 \\
\hline \multicolumn{3}{|l|}{ Local } \\
\hline CAPS $^{*}$ & 6 & 3,87 \\
\hline Hospital & 5 & 3,22 \\
\hline Centro de Convivência & 4 & 2,58 \\
\hline $\mathrm{SRT}^{* *}$ & 112 & 72,25 \\
\hline $\mathrm{CIJ}^{\star * *}$ & 7 & 4,51 \\
\hline $\mathrm{NESM}^{* * * *}$ & 21 & 13,54 \\
\hline \multicolumn{3}{|l|}{ Faixa etária dos filhos } \\
\hline Sem filhos & 27 & 17,4 \\
\hline Filhos até 6 anos & 24 & 15,5 \\
\hline Filhos entre 6 e 18 anos & 57 & 36,8 \\
\hline Filhos acima de 18 anos & 45 & 29 \\
\hline S/Inf. & 2 & 1,3 \\
\hline \multicolumn{3}{|c|}{ Uso de serviços de saúde mental } \\
\hline Sim & 60 & 38,7 \\
\hline Não & 92 & 59,4 \\
\hline S/Inf. & 3 & 1,9 \\
\hline
\end{tabular}

${ }^{*}$ Centro de Atenção Psicossocial; ${ }^{*}$ Serviço de Residência Terapêutica; ${ }^{* * *}$ Centro de Atenção para Infância e Juventude; ${ }^{* * *}$ Núcleo Estadual em Saúde Mental do que $30 \%$ de atitudes negativas: itens 3, 6 e 8 da subescala Autoritarismo e item 10 da subescala Restrição Social (Tabela 2).

Como podemos perceber na Tabela 3 , três das dez características sociodemográficas (categoria profissional, nível educacional e faixa etária) mostraram diferenças significativas nas atitudes dos trabalhadores nas quatro subescalas.

$\mathrm{Na}$ análise bivariada, encontramos que os indivíduos com filhos entre 6 e 18 anos tiveram maior possibilidade de ter atitudes negativas na subescala Autoritarismo $(\mathrm{F}=3,629 ; \mathrm{p}=0,014)$ do que os que não tinham filhos. Os profissionais com faixa etária acima de 60 anos apresentaram atitudes significativamente mais negativas quando comparados aos da faixa etária entre 20 a 30 anos nas subescalas Autoritarismo ( $\mathrm{F}=3,207 ; \mathrm{p}=0,015)$ e Restrição Social $(\mathrm{F}=2,647 ; \mathrm{p}=0,036)$.

Na subescala Autoritarismo, ter mais de 12 anos de estudo foi preditor de atitudes mais positivas em relação a todas as outras categorias ( $\mathrm{F}=17,828 ; \mathrm{p}=0,000)$; na subescala Restrição Social, ter mais de 12 anos de estudo foi preditor de atitudes mais positivas quanto aos que tinham menos de 9 anos de estudo ( $\mathrm{F}=8,366 ; \mathrm{p}=0,000)$; na subescala Benevolência, ter mais de 10 anos de estudo foi preditor de atitudes mais positivas do que aqueles com menos de 10 anos de estudo ( $F=10,599 ; \mathrm{p}=0,000)$; e na subescala Ideologia da Saúde Mental Comunitária, ter mais de 12 anos de estudo foi preditor de atitudes mais positivas em comparação com todas as outras categorias $(\mathrm{F}=8,967 ; \mathrm{p}=0,000)$.

Os funcionários com categoria de Cuidador e Administrativo apresentaram atitudes significativamente mais negativas na subescala Autoritarismo $(\mathrm{F}=13,104 ; \mathrm{p}=0,000)$ do que aqueles com função de Nível Superior. Os funcionários da categoria de Cuidador mantiveram essa diferença quando comparados aos funcionários da categoria de Coordenação na subescala Restrição Social $(\mathrm{F}=9,680 ; \mathrm{p}=0,004)$ e a todos as outras categorias na subescala Ideologia da Saúde Mental Comunitária $(\mathrm{F}=6,832 ; \mathrm{p}=0,000)$ (Tabela 3).

\section{DISCUSSÃO}

A maioria dos trabalhadores em saúde mental da cidade de Carmo apresentou atitudes positivas com relação aos indivíduos com transtornos mentais nas quatro subescalas. Essas atitudes positivas foram estatisticamente significativas em três características sociodemográficas: nível educacional, categoria profissional e faixa etária.

$\mathrm{O}$ estudo mostrou uma associação significativa entre o nível de escolaridade e as atitudes em relação aos pacientes psiquiátricos, com destaque para as pessoas com mais de 12 anos de estudo, que tiveram atitudes mais positivas em todas as subescalas. Esse resultado corrobora os achados de Avanci et al. ${ }^{18}$ e Baker e Schulberg ${ }^{19}$.

Os profissionais que apresentaram atitudes mais positivas para com os pacientes foram os trabalhadores que exerciam 
Tabela 2. Frequência de atitudes negativas e positivas na população estudada ( $n=155)$

\section{Autoritarismo}

1. Uma das principais causas da doença mental é a falta de autodisciplina e autocontrole.

2. A melhor forma de controlar os doentes mentais é mantê-los trancados.

3. Algumas características diferenciam os doentes mentais das pessoas normais.

4. Uma pessoa sempre deve ser hospitalizada assim que apresentar sinais de distúrbio mental.

5. Doentes mentais precisam do mesmo tipo de controle e disciplina que as crianças pequenas.

6. Doença mental é uma doença como outra qualquer.

7. Os doentes mentais não deveriam ser tratados como indesejáveis na sociedade.

8. Deveria ser dada menos importância em proteger a sociedade dos doentes mentais.

9. Os hospitais psiquiátricos são um modelo ultrapassado de tratamento para os doentes mentais.

10. Qualquer um pode se tornar um doente mental.

Restrição social

1. Nenhuma responsabilidade deveria ser dada ao doente mental.

2. Os doentes mentais deveriam ser isolados do resto da comunidade.

3. Uma mulher seria tola em se casar com um homem que tenha sofrido de doença mental, mesmo que ele parecesse completamente recuperado.

4. Eu não gostaria de ser vizinho de porta de alguém que tenha sido doente mental.

5. Alguém que tenha uma história de doença mental deveria ser impedido de assumir qualquer emprego público.

6. Os doentes mentais não deveriam ser privados de seus direitos individuais.

7. Doentes mentais deveriam ser encorajados a assumir responsabilidades de vida normal.

8. Ninguém tem o direito de excluir os doentes mentais de sua vizinhança.

9. Os doentes mentais oferecem menos perigo do que a maioria das pessoas pensa.

10. A maior parte das mulheres que foram pacientes de um hospital psiquiátrico pode ser confiável como babá.

Benevolência

1. Os doentes mentais foram, por muito tempo, objetos de ridicularização.

2. Deveriam ser gastos mais recursos públicos no cuidado e tratamento dos doentes mentais.

3. Nós precisamos ser mais tolerantes com os doentes mentais na nossa sociedade.

4. Nossos hospitais psiquiátricos parecem mais prisões do que lugares para se cuidar dos doentes mentais.

5. A sociedade tem a responsabilidade de fornecer o melhor tratamento possível aos doentes mentais.

6. Os doentes mentais não merecem nossa simpatia.

7. Os doentes mentais são um peso para a sociedade.

8. Aumentar as despesas com saúde mental é um desperdício de recursos públicos.

9. Existem serviços de atendimento suficientes para os doentes mentais.

10. É melhor evitar alguém que tenha problemas mentais.

Ideologia da Saúde Mental Comunitária

1. Todos deveriam aceitar que serviços de atenção à saúde mental fossem instalados na sua vizinhança.

2. A melhor terapia para muitos doentes mentais é fazer parte de uma comunidade normal

3. A assistência em saúde mental deveria ser oferecida, na medida do possível, em serviços localizados na comunidade.

4. A localização de serviços de saúde mental em bairro residencial não coloca em perigo os moradores.

5. Moradores não têm nada a temer de pessoas que venham à sua vizinhança para receber atendimento em saúde mental.

6. Serviços de saúde mental deveriam ser mantidos longe de bairros residenciais.

7. Moradores locais têm boas razões para resistir à implantação de serviços de saúde mental na sua vizinhança.

8. Morar em áreas residenciais pode ser uma boa terapia para os pacientes, mas o risco para os moradores é muito grande.

9. É assustador pensar em pessoas com doença mental morando em bairros residenciais.

10. Localizar serviços de saúde mental em área residencial desvaloriza o bairro.

\begin{tabular}{|c|c|c|}
\hline Negativa & Indiferente & Positiva \\
\hline 17,4 & 16,1 & 66,5 \\
\hline 1,3 & 4,5 & 94,2 \\
\hline 56,8 & 11,6 & 31,6 \\
\hline 24,5 & 12,9 & 62,6 \\
\hline 20,6 & 16,2 & 63,2 \\
\hline 47,7 & 11,0 & 41,3 \\
\hline 17,4 & 3,2 & 79,4 \\
\hline 34,8 & 8,4 & 56,8 \\
\hline 12,9 & 1,9 & 85,2 \\
\hline 3,2 & 3,9 & 92,9 \\
\hline 10,3 & 14,2 & 75,5 \\
\hline 2,6 & 3,2 & 94,2 \\
\hline 11,6 & 14,2 & 74,2 \\
\hline 2,6 & 7,1 & 90,3 \\
\hline 5,8 & 9,0 & 85,2 \\
\hline 20,6 & 3,9 & 75,5 \\
\hline 5,8 & 1,3 & 92,9 \\
\hline 3,2 & 1,9 & 94,8 \\
\hline 5,8 & 4,5 & 89,7 \\
\hline 40,0 & 23,2 & 36,8 \\
\hline 9,0 & 2,6 & 88,4 \\
\hline 1,3 & 1,3 & 97,4 \\
\hline 3,2 & 2,6 & 94,2 \\
\hline 8,4 & 3,2 & 88,4 \\
\hline 3,2 & 5,8 & 91,0 \\
\hline 3,2 & 3,9 & 92,9 \\
\hline 3,2 & 5,2 & 91,6 \\
\hline 2,6 & 4,5 & 92,9 \\
\hline 19,4 & 9,7 & 71,0 \\
\hline 3,2 & 4,5 & 92,3 \\
\hline 3,9 & 3,2 & 92,9 \\
\hline 1,9 & 1,9 & 96,2 \\
\hline 0,0 & 0,6 & $\mathbf{9 9 , 4}$ \\
\hline 20,0 & 3,2 & 76,8 \\
\hline 6,5 & 6,4 & 87,1 \\
\hline 1,3 & 1,9 & 96,8 \\
\hline 8,4 & 5,1 & 86,5 \\
\hline 7,7 & 5,8 & 86,5 \\
\hline 0,6 & 5,2 & 94,2 \\
\hline 3,9 & 7,1 & 89,0 \\
\hline
\end{tabular}


Tabela 3. Relação entre os escores da escala CAMI e os dados sociodemográficos (MANOVA)

\begin{tabular}{|c|c|c|c|c|c|c|c|c|c|c|}
\hline \multirow{2}{*}{ Variável } & \multicolumn{2}{|c|}{ Autoritarismo } & \multicolumn{2}{|c|}{ Restrição Social } & \multicolumn{2}{|c|}{ Benevolência } & \multicolumn{2}{|c|}{$\begin{array}{l}\text { Ideologia da } \\
\text { Comunidade }\end{array}$} & \multirow{2}{*}{$\mathbf{F}$} & \multirow{2}{*}{ p-valor } \\
\hline & $\mathbf{N}$ & Média (dp) & $\mathbf{N}$ & Média (dp) & $\mathbf{N}$ & $\begin{array}{l}\text { Média } \\
(\text { dp) }\end{array}$ & $\mathbf{N}$ & Média (dp) & & \\
\hline \multicolumn{11}{|l|}{ Sexo } \\
\hline Feminino & 111 & $2.69(0,51)$ & 111 & $3,03(0,41)$ & 111 & $3,24(0,43)$ & 111 & $3,23(0,42)$ & \multirow[b]{2}{*}{1,398} & \multirow{2}{*}{0,24} \\
\hline Masculino & 44 & $2,58(0,48)$ & 44 & $3,09(0,56)$ & 44 & $3,33(0,42)$ & 44 & $3,29(0,53)$ & & \\
\hline \multicolumn{11}{|l|}{ Estado civil } \\
\hline Solteiro & 40 & $2,76(0,45)$ & 40 & $3,03(0,46)$ & 40 & $3,26(0,36)$ & 40 & $3,29(0,41)$ & \multirow{5}{*}{1,394} & \multirow{5}{*}{0,14} \\
\hline Casado & 94 & $2,61(0,50)$ & 94 & $3,07(0,46)$ & 94 & $3,32(0,42)$ & 94 & $3,23(0,46)$ & & \\
\hline Viúvo & 4 & $2,58(0,13)$ & 4 & $3,05(0,34)$ & 4 & $2,83(0,36)$ & 4 & $2,90(0,29)$ & & \\
\hline Separado & 5 & $2,54(0,39)$ & 5 & $2,98(0,43)$ & 5 & $2,98(0,82)$ & 5 & $3,22(0,59)$ & & \\
\hline Divorciado & 12 & $2,80(0,51)$ & 12 & $3,03(0,59)$ & 12 & $3,23(0,45)$ & 12 & $3,35(0,46)$ & & \\
\hline \multicolumn{9}{|l|}{ Categoria } & \multirow[t]{5}{*}{4,151} & \multirow[t]{5}{*}{$<0,01$} \\
\hline Cuidador & 112 & $2,53(0,45)$ & 112 & $2,98(0,45)$ & 112 & $3,20(0,44)$ & 112 & $3,15(0,43)$ & & \\
\hline $\begin{array}{l}\text { Função } \\
\text { Administrativa }\end{array}$ & 12 & $2,65(0,58)$ & 12 & $3,17(0,55)$ & 12 & $3,47(0,38)$ & 12 & $3,52(0,45)$ & & \\
\hline $\begin{array}{l}\text { Função de Nível } \\
\text { Superior }\end{array}$ & 25 & $3,13(0,41)$ & 25 & $3,23(0,38)$ & 25 & $3,42(0,38)$ & 25 & $3,44(0,40)$ & & \\
\hline Coordenação & 6 & $3,00(0,37)$ & 6 & $3,50(0,37)$ & 6 & $3,53(0,23)$ & 6 & $3,65(0,31)$ & & \\
\hline \multicolumn{11}{|l|}{ Educação } \\
\hline$\leq 9$ anos & 50 & $2,42(0,46)$ & 50 & $2,88(0,42)$ & 50 & $3,07(0,41)$ & 50 & $3,09(0,43)$ & \multirow{3}{*}{6,008} & \multirow{3}{*}{$<0,01$} \\
\hline 10 a 12 anos & 55 & $2,60(0,41)$ & 55 & $3,06(0,47)$ & 55 & $3,31(0,44)$ & 55 & $3,23(0,45)$ & & \\
\hline$>12$ anos & 48 & $2,96(0,50)$ & 48 & $3,24(0,42)$ & 48 & $3,44(0,36)$ & 48 & $3,44(0,39)$ & & \\
\hline \multicolumn{11}{|l|}{ Faixa etária dos filhos } \\
\hline Sem filhos & 27 & $2,87(0,41)$ & 27 & $3,17(0,55)$ & 27 & $3,27(0,34)$ & 27 & $3,29(0,42)$ & \multirow{4}{*}{1,576} & \multirow{4}{*}{0,09} \\
\hline $\begin{array}{l}\text { Pelo menos um filho } \\
\text { menor que } 6 \text { anos }\end{array}$ & 24 & $2,75(0,52)$ & 24 & $3,10(0,41)$ & 24 & $3,44(0,38)$ & 24 & $3,30(0,45)$ & & \\
\hline $\begin{array}{l}\text { Somente filhos entre } \\
6 \text { e } 18 \text { anos }\end{array}$ & 57 & $2,52(0,52)$ & 57 & $3,04(0,51)$ & 57 & $3,22(0,40)$ & 57 & $3,22(0,47)$ & & \\
\hline $\begin{array}{l}\text { Somente filhos acima } \\
\text { de } 18 \text { anos }\end{array}$ & 45 & $2,68(0,47)$ & 45 & $2,99(0,39)$ & 45 & $3,26(0,52)$ & 45 & $3,25(0,43)$ & & \\
\hline \multicolumn{11}{|l|}{ Utilização serviço } \\
\hline Sim & 60 & $2,60(0,53)$ & 60 & $3,03(0,45)$ & 60 & $3,29(0,44)$ & 60 & $3,23(0,45)$ & \multirow{3}{*}{0,995} & \\
\hline Não & 92 & $2,75(0,46)$ & 92 & $3,10(0,49)$ & 92 & $3,24(0,42)$ & 92 & $3,28(0,45)$ & & 0,44 \\
\hline S/Inf. & 3 & $2,73(0,15)$ & 3 & $2,87(0,15)$ & 3 & $3,23(0,25)$ & 3 & $3,23(0,46)$ & & \\
\hline Imóvel & & & & & & & & & & \\
\hline Próprio & 105 & $2,63(0,50)$ & 105 & $3,04(0,47)$ & 105 & $3,26(0,47)$ & 105 & $3,27(0,46)$ & & \\
\hline Alugado & 44 & $2,71(0,52)$ & 44 & $3,08(0,46)$ & 44 & $3,32(0,35)$ & 44 & $3,19(0,45)$ & 0,707 & 0,685 \\
\hline S/Inf. & 6 & $2,72(0,42)$ & 6 & $3,08(0,31)$ & 6 & $3,18(0,26)$ & 6 & $3,19(0,34)$ & & \\
\hline Faixa Etária & & & & & & & & & & \\
\hline 20 a 30 & 20 & $2,85(0,58)$ & 20 & $3,22(0,48)$ & 20 & $3,39(0,34)$ & 20 & $3,45(0,43)$ & & \\
\hline 31 a 40 & 51 & $2,69(0,46)$ & 51 & $3,10(0,53)$ & 51 & $3,20(0,39)$ & 51 & $3,16(0,44)$ & & \\
\hline 41 a 50 & 51 & $2,56(0,47)$ & 51 & $3,03(0,36)$ & 51 & $3,27(0,40)$ & 51 & $3,21(0,42)$ & 1,955 & 0,02 \\
\hline 51 a 60 & 27 & $2,74(0,53)$ & 27 & $2,97(0,41)$ & 27 & $3,38(0,48)$ & 27 & $3,34(0,44)$ & & \\
\hline$>60$ & 6 & $2,13(0,36)$ & 6 & $2,60(0,41)$ & 6 & $3,07(0,81)$ & 6 & $3,13(0,68)$ & & \\
\hline
\end{tabular}

função de Coordenação, seguidos pelos trabalhadores com funções de Nível Superior ${ }^{6,8}$. Em geral, os trabalhadores de saúde mental de Carmo se mostraram comprometidos com as funções que exerciam, o que pode ser evidenciado pela alta frequência das atitudes positivas nas subescalas de Ideologia da Saúde Mental Comunitária e Benevolência, beneficiando a inserção social e o cuidado comunitário dos pacientes, fatores que são cruciais no processo da desinstitucionalização psiquiátrica $^{20}$.

Os profissionais com idade entre 20 e 30 anos tiveram atitudes mais positivas em comparação às pessoas com mais de 60 anos. As pessoas mais jovens começaram a trabalhar 
em um momento em que a implementação da reforma da saúde mental já estava sendo estabelecida, enquanto que os trabalhadores de saúde mental com idade mais avançada, na sua maioria, conviveram durante muito tempo com uma ideologia de exclusão social dos pacientes, representada pelo hospital colônia 5 .

Os Cuidadores apresentaram as atitudes mais negativas nas subescalas Autoritarismo, Restrição Social e Ideologia da Saúde Mental Comunitária em comparação aos outros funcionários. Esses profissionais têm um contato mais permanente com os moradores das residências terapêuticas, e esse dado é importante para analisar a relação contato e atitudes negativas. Os Cuidadores convivem diariamente e vivenciam diretamente as dificuldades apresentadas pelos pacientes com transtornos mentais graves.

Nos achados de autores como Thorpe e Addison ${ }^{7}$, Adewuya e Makanjuola ${ }^{21}$ e Boyd et al. ${ }^{22}$, o contato teve uma importante associação com as atitudes positivas. Pelo contrário, Gaebel et al..$^{23}$, na Alemanha, demonstraram que as atitudes negativas e a distância social aumentaram com um maior contato com os pacientes

Avanci et al. ${ }^{18}$ encontraram que o contato influenciava nas atitudes positivas quando era acompanhado de conhecimento técnico. Esse achado coincide com as conclusões do estudo de Eack e Newhill ${ }^{24}$, que identificaram uma relação entre o contato e a experiência e conhecimento técnico com uma atitude mais positiva para com os indivíduos com transtornos mentais.

A subescala Ideologia da Saúde Mental Comunitária diz respeito a vários aspectos importantes para o processo da desinstitucionalização psiquiátrica, como: o impacto da implantação dos serviços de saúde mental, o potencial perigo que o doente mental representaria para os vizinhos e a aceitação dos princípios da desinstitucionalização psiquiátrica. Essa subescala teve altas frequências de atitudes positivas no presente estudo, o que pode ser reflexo da implementação dos SRT, um dos eixos principais da política de saúde mental do município.

Segundo o relato de um supervisor dos SRT, quando começou o projeto de implantação das residências, as pessoas da cidade rejeitavam os pacientes egressos do hospital colônia, porém, atualmente, já aceitam que eles aluguem suas casas na vizinhança. Por se tratar de uma cidade pequena, o intercâmbio comercial com os pacientes é um fator importante para a inclusão deles na comunidade. Essa inclusão, em alguns casos, parece fazer com que a população do município tenha determinados comportamentos para com eles. Por exemplo, um paciente falava de uma situação na qual discutiu com a dona de um bar porque ela se recusou a lhe vender bebida alcoólica. Essa situação pode ser interpretada como cuidado, mas também como controle e restrição da comunidade com esses pacientes.

O presente estudo não encontrou associação estatisticamente significativa entre o uso de serviços em saúde mental e atitudes dos profissionais. Trata-se de um resultado diferente de outras pesquisas, como a de Angermeyer et al. ${ }^{25}$, que identificaram associação entre o conhecimento de serviços de saúde mental por utilização própria ou por tratamento de familiares ou amigos e as atitudes em relação aos pacientes. Para autores como Schafer et al. ${ }^{3}$, conhecer alguma pessoa que tenha sofrido de doença mental, assim como trabalhar em saúde mental, influencia nas atitudes positivas para com os pacientes com transtornos mentais. Entretanto, no presente estudo, foram analisados profissionais que lidam diariamente com o paciente, o que pode ter influenciado o resultado.

Para que um processo de desinstitucionalização psiquiátrica seja bem-sucedido, é preciso a existência de alguns elementos-chave: estabelecimento de uma comunidade compreensiva que ofereça suporte; ambiente que permita a troca de experiência da população com os pacientes; respeito aos direitos dos pacientes como cidadãos; uma comunidade que não discrimine ${ }^{1}$. O pré-requisito para estabelecimento de serviços de cuidado na comunidade é ter uma população que cuide ${ }^{26}$.

\section{LIMITAÇÕES DO ESTUDO}

1. Trata-se de um estudo transversal, e, portanto, os resultados podem sofrer viés temporal;

2. Os resultados podem não ser representativos de outras populações em função da especificidade da população estudada.

\section{CONCLUSÃO}

A ressocialização e a recuperação de pacientes com transtornos mentais dependem da aceitação e do estímulo dos trabalhadores de saúde, principalmente dos trabalhadores de saúde mental. Este estudo demonstrou que os trabalhadores de saúde mental do município de Carmo apresentaram atitudes preponderantemente positivas em relação aos pacientes. Esse resultado foi possivelmente influenciado pelas políticas de saúde mental implementadas nos últimos 15 anos em Carmo, que tiveram como resultado não somente a criação de novos dispositivos de atenção em saúde mental, mas, principalmente, a inclusão dos moradores do antigo hospital colônia na comunidade.

Entretanto, é importante que programas educacionais continuem a ser implementados não só na cidade de Carmo, mas se estendam a outros municípios. 


\section{REFERÊNCIAS}

1. Hannigan B. Mental health care in the comunity: an analysis of a contemporary public attitude towards and public representations of mental illness. J Ment Health. 1999;8(5):431-40. http://dx.doi.org/10.1080/09638239917148.

2. Rössler W, Salize H. Factors affecting public attitudes towards mental health care. Eur Arch Psychiatry Clin Neurosci. 1995;245(1):20-6. PMid:7786908. http://dx.doi.org/10.1007/BF02191540.

3. Schafer T, Wood S, Williams R. A survey into student nurses' attitudes towards mental illness: Implications for nurse trainning. Nurse Educ Today. 2011;31(4):328-32. PMid:20655630. http://dx.doi.org/10.1016/j. nedt.2010.06.010

4. Chambers M, Guise V, Välimäki M, Botelho MA, Scott A, Staniuliené $\mathrm{V}$, et al. Nurses attitudes to mental illness: a comparison of a sample of nurses from five European countries. Int J Nurs Stud. 2010;47(3):350-62. PMid:19804882. http://dx.doi.org/10.1016/j.ijnurstu.2009.08.008.

5. Siqueira SRG, Abelha L, Lovisi GM, Sarução KR, Yang L. Attitudes towards mentally Ill: a study with health workers at a University Hospital in Rio de Janeiro. Psychiatr Q. 2017;88(1):25-38. PMid:26951160. http://dx.doi. org/10.1007/s11126-016-9431-5.

6. Nordt C, Rossler W, Lauber C. Attitudes of mental health professionals towards people with schizophrenia and major depression. Schizophr Bull. 2006;32(4):709-14. PMid:16510695. http://dx.doi.org/10.1093/schbul/ sbj065.

7. Thorpe SJ, Addison SJ. Factors involved in the formation of attitudes towards those who are mentally ill. Soc Psychiatry Psychiatr Epidemiol. 2004;39(3):22834. PMid:14999456. http://dx.doi.org/10.1007/s00127-004-0723-y.

8. Lauber C, Anthony M, Ajdacic-Gross V, Rössler W. Evaluating explicit and implicit estigma on mental illness in mental health profissionals and medical students. Community Ment Health J. 2015;51(5):628-34. PMid:25535045. http://dx.doi.org/10.1007/s10597-014-9796-6.

9. Lauber C, Anthony M, Ajdacic-Gross V, Rössler W. What about psychiatrys attitude to mentally ill people? Eur Psychiatry. 2004;19(7):423-7. PMid:15504649. http://dx.doi.org/10.1016/j.eurpsy.2004.06.019.

10. Aydin N, Yigit A, Inandi T, Kirpinar I. Attitudes of hospital staff toward mentally ill patients in a teaching hospital in Turkey. Int J Soc Psychiatry. 2003;49(1):17-26. PMid:12793512. http://dx.doi.org/10.1177/002076400 3049001544 .

11. Sévigny R, Yang W, Zhang P, Marleau JD, Yang Z, Su L, et al. Attitudes towards mentally ill in a sample of professionals working in a psychiatric hospital in Beiing, China. Int J Soc Psychiatry. 1999;45(1):41-55. PMid:10443248. http://dx.doi.org/10.1177/002076409904500106.

12. Loch AA. Stigma and higher rates of psychiatric hospitalitation: Sao Paulo public mental health system. Rev Bras Psiquiatr. 2012;34(2):185-92. PMid:22729415. http://dx.doi.org/10.1590/S1516-44462012000200011.

13. Instituto Brasileiro de Geografia e Estatística. IBGE Cidades: Carmo [Internet]. Rio de Janeiro: IBGE; 2016 [citado em 2017 fev 28]. Disponível em: http://cidades.ibge.gov.br/xtras/perfil.php?codmun $=330120$

14. Taylor SM, Dear M. Scaling comunity attitudes toward the mentally ill. Schizophr Bull. 1981;7(2):225-40. PMid:7280561.http://dx.doi.org/10.1093/schbul/7.2.225.
15. Gonçalves S, Abelha L, Legay LF, Lovisi GM. Equivalência conceitual e semântica da versão em português da escala Comunity Attitude Toward Mentally Ill (CAMI-BR). Cad Saude Colet. 2008;16(4):749-64.

16. Wolff G, Pathare S, Craig T, Leff J. Community attitudes to mental illness. $\mathrm{Br}$ J Psychiatry. 1996;168(2):183-90. PMid:8837908. http://dx.doi.org/10.1192/ bjp.168.2.183.

17. Tabachnick BG, Fidell LS. Using multivariate statistics. Northridge: California State University; 2001.

18. Avanci RC, Malaguti SE, Pedrão LJ. Autoritarismo e benevolência frente à doença mental: estudo com alunos ingressantes no curso de enfermagem. Rev Lat Am Enfermagem. 2002;10(4):509-15. PMid:12592851. http://dx.doi. org/10.1590/S0104-11692002000400007.

19. Baker F, Schulberg HC. The development of a comunity mental health ideology scale. Community Ment Health J. 1967;3(3):216-25. PMid:24186602. http://dx.doi.org/10.1007/BF01434874.

20. Song LY, Chang LY, Shih CY, Lin CY, Yang MJ. Community attitudes towards the mentally ill: the results of a national survey of the Taiwanese population. Int J Soc Psychiatry. 2005;51(2):162-76. PMid:16048245. http:// dx.doi.org/10.1177/0020764005056765.

21. Adewuya A, Makanjuola R. Social distance towards people with mental illness amongst Nigerian university students. Soc Psychiatry Psychiatr Epidemiol. 2005;40(11):865-8. PMid:16234984. http://dx.doi.org/10.1007/ s00127-005-0965-3.

22. Boyd JE, Katz EP, Link BG, Phelan JC. The relationship of multiple aspects of stigma and personal contact with someone hospitalized for mental illness in a nationally representative sample. Soc Psychiatry Psychiatr Epidemiol. 2010;45(11):1063-70. PMid:19823756. http://dx.doi.org/10.1007/s00127009-0147-9.

23. Gaebel W, Baumann A, Witte AM, Zaeske H. Public attitudes towards people with mental illness in six German cities: results of a public survey under special consideration of schizophrenia. Eur Arch Psychiatry Clin Neurosci. 2002;252(6):278-87. PMid:12563536. http://dx.doi.org/10.1007/ s00406-002-0393-2.

24. Eack SM, Newhill CE. An investigation of the relations between student knowledge, personal contact and attitudes toward individuals with schizophrenia. J Soc Work Educ. 2008;44(3):1-18. PMid:24353396. http:// dx.doi.org/10.5175/JSWE.2008.200700009.

25. Angermeyer MC, Matschinger H, Corrigan PW. Familiarity with mental illness and social distance from people with schizophrenia and major depression: testing a model using data from a representative population survey. Schizophr Res. 2004;69(2-3):175-82. PMid:15469191. http://dx.doi. org/10.1016/S0920-9964(03)00186-5.

26. Bhugra D. Attitudes towards mental illness: a review of the literature. Acta Psychiatr Scand. 1989;80(1):1-12. PMid:2669442. http://dx.doi. org/10.1111/j.1600-0447.1989.tb01293.x. 